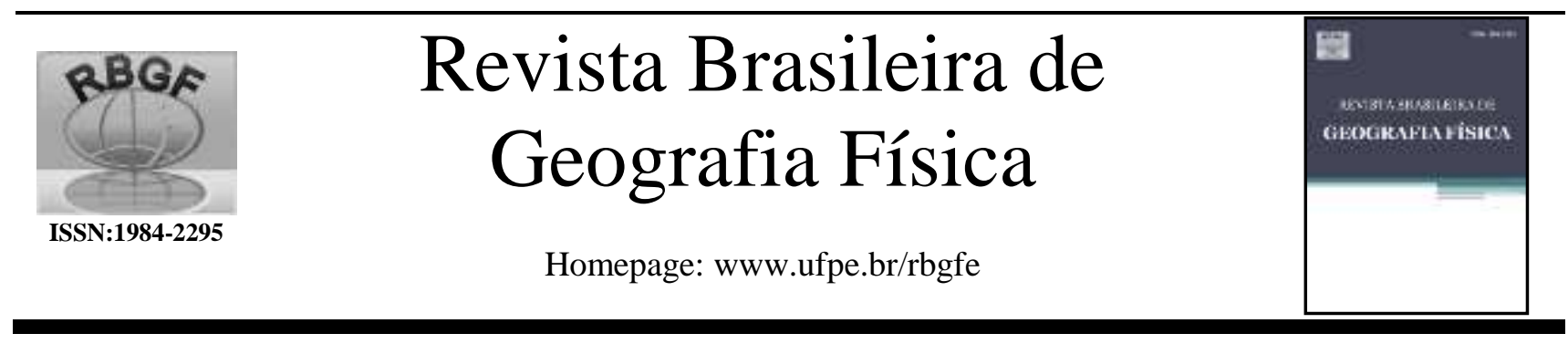

\title{
EXPANSÃO DA AGRICULTURA IRRIGADA E MUDANÇAS NOS PROCESSOS DE INTERAÇÃO SUPERFÍCIE-ATMOSFERA: UM ESTUDO NUMÉRICO DE IMPACTO AMBIENTAL EM ÁREAS DE CAATINGA
}

\author{
Ewerton Cleudson de Sousa Melo $^{1}$, Magaly de Fatima Correia ${ }^{2}$, Maria Regina da Silva Aragão ${ }^{2}$ \\ ${ }^{1}$ Pesquisador, Universidade Federal de Campina Grande, Campina Grande, PB, Brasil; e-mail:ecsmelo@ yahoo.com. ${ }^{2}$ Professora, Departamento de \\ Ciências Atmosféricas da UFCG, Campina Grande, PB, Brasil; e-mail: magaly@dca.ufcg.edu.br; regina@dca.ufcg.edu.br
}

Artigo recebido em 09/11/2011 e aceito em 27/12/2011

\begin{abstract}
R E S U M O
O RAMS (Sistema de Modelagem Atmosférica Regional) na versão 8.0 é utilizado neste estudo com o objetivo de avaliar como a expansão da agricultura irrigada pode modificar processos de interação entre a superfície e a atmosfera. Dois cenários (com e sem influência antrópica) são utilizados para simular mudanças na configuração espacial dos fluxos turbulentos em condições atmosféricas moduladas pela atuação de um VCAN (vórtice ciclônico de altos níveis). Os resultados indicam que a variabilidade espacial dos fluxos turbulentos é altamente influenciada pelas características da superfície, topografia, umidade no solo e tipo de vegetação. Por outro lado, a redução no teor de umidade da atmosfera, normalmente referenciado como déficit de pressão de vapor (DPV), é outro fator que exerce um forte controle sobre a taxa de evapotranspiração e pode alterar consideravelmente o balanço de energia em superfície. Nos períodos de atividade convectiva intensa a influência da irrigação nos fluxos turbulentos não é evidente. Em contrapartida, os fluxos simulados nos dias sem precipitação são bastante afetados pela disponibilidade de água no solo nos perímetros irrigados. Esse efeito é mais pronunciado do que o da mudança das áreas de vegetação nativa por áreas agrícolas.
\end{abstract}

Palavras-chave: RAMS, uso da terra, irrigação, microclima, caatinga.

\section{EXPANSION OF IRRIGATED AGRICULTURE AND CHANGES IN PROCESSES OF SURFACE-ATMOSPHERE INTERACTION: A NUMERICAL STUDY OF ENVIRONMENTAL IMPACT ON CAATINGA AREAS}

A B S T R A C T

The RAMS (Regional Atmospheric Modeling System) version 8.0 is used in this study aiming to evaluate how the expansion of the irrigated agriculture can modify the processes of interaction between the surface and the atmosphere. Two scenarios (with and without anthropogenic influence) are used to simulate changes in the spatial pattern of the turbulent fluxes in atmospheric conditions modulated by an ULCV (upper level cyclonic vortex). The results indicate that the spatial variability of the turbulent fluxes is highly influenced by surface characteristics, topography, soil moisture and vegetation type. On the other hand, a reduction in the moisture content of the atmosphere, normally referenced as the vapor pressure deficit (VPD), is another factor that exerts a strong control upon the evapotranspiration rate and may considerably alter the surface energy balance. In periods of intense convective activity the influence of irrigation upon the turbulent fluxes is not evident. By contrast, the fluxes simulated in rainless days are highly affected by the availability of water in the soil of the irrigated perimeters. This effect is more pronounced than the one due to the change of the native vegetation areas by agricultural areas..

* E-mail para correspondência: ecsmelo@yahoo.com

(Melo, E. C. S.). 


\section{Introdução}

Descontinuidades na cobertura vegetal resultantes da implantação de perímetros irrigados causam mudanças físicas importantes como alterações no albedo, na rugosidade da superfície e na umidade do solo. Essas mudanças são capazes de provocar modificações complexas no balanço de energia em superfície e consequentemente na estrutura da CLP (camada limite planetária). Observa-se geralmente, aumento da energia total disponível, com aumento do fluxo de calor latente resultante dos processos de evapotranspiração e geração de circulações em mesoescala (Adegoke et al., 2007).

A irrigação normalmente contribui com o aumento da precipitação quando mecanismos dinâmicos em grande escala favorecem a convergência em baixos níveis e movimentos ascendentes, possibilitando que o aumento da umidade proporcionada pela irrigação chegue à base das nuvens.

No final da década de 70, o semiárido da BHRS (bacia hidrográfica do rio São Francisco), particularmente na área referenciada como eixo Petrolina-Juazeiro, passou por modificações marcantes na cobertura do solo e o uso da terra. A construção da Usina de Sobradinho e a crescente expansão da agricultura irrigada mudou substancialmente a paisagem da região. Estas mudanças motivaram a realização de vários estudos com o objetivo de quantificar o impacto das mudanças antrópicas no clima local (Correia, 2001; Correia \& Silva Dias, 2003; Correia et al., 2006a,b). Modelagem numérica e a aplicação de técnicas estatísticas foram utilizadas como principais ferramentas de análise.

Os resultados mostram que o vento, a temperatura e a umidade do ar estão entre os elementos climáticos mais afetados pelas mudanças ambientais na região (Correia, 2001; Correia \& Silva Dias, 2003; Correia et al., 2006a,b; Melo, 2011). A descontinuidade na cobertura do solo deu origem a circulações induzidas termicamente como a brisa lacustre. As camadas mais baixas da atmosfera também foram impactadas pela mudança no tipo da vegetação. Circulações induzidas por descontinuidades entre áreas irrigadas e vegetação do tipo Caatinga contribuíram com a intensificação da brisa lacustre atingindo extensões maiores no entorno da represa. As análises mostraram ainda que a topografia é um fator importante na geração de circulações locais na região. Foi possível verificar, através de diferentes simulações, que a interação entre circulações induzidas pela topografia e pelo lago, aliada à presença ou ausência do vento sinótico, pode gerar mecanismos dinâmicos que afetam o tempo local.

Estudos sobre o efeito da irrigação na precipitação desenvolvido por Barnston e Schickedanz, (1984) indicam que o grau de estabilidade da atmosfera é um fator importante nos processos de transferência vertical do vapor. Situações em que a taxa de variação vertical da temperatura do ar é estável, o excesso de umidade geralmente não ascende mais do que 10 a 20 $\mathrm{m}$ acima da superfície. Em períodos caracterizados pela sequência de dias quentes e secos, a umidade adicional oriunda da evaporação não atinge o nível de condensação. Grande parte da umidade é transportada horizontalmente por ventos sinóticos persistentes à superfície. No entanto, em condições instáveis (taxa de variação vertical da temperatura do ar maior do que a taxa adiabática seca), qualquer parcela de ar que ascenda na atmosfera continuará a subir até que sua taxa de variação vertical da temperatura se estabilize o suficiente. Nessas situações, qualquer umidade adicional nos baixos níveis tem o potencial para produzir maior conteúdo de água nas nuvens e maiores volumes de chuva.

Outros estudos mostram que o resfriamento à superfície produzido por irrigação é acompanhado por aumento no fluxo de calor latente (fluxo de vapor d'água) em camadas de até $500 \mathrm{~m}$ acima da superfície, redução no fluxo de calor sensível e aumento na temperatura do ponto de orvalho (Adegoke et. al., 2003; Stolhgren et al,. 1998). A temperatura do ponto de orvalho elevada e fluxos de umidade na camada limite planetária podem aumentar a energia convectiva disponível, aumentando a instabilidade atmosférica e a cobertura de nuvens no período diurno.

Em síntese, um aumento da umidade em superfície reduz o fluxo de calor sensível, enquanto aumenta a transferência de água para a atmosfera por meio da evaporação e da transpiração (Pielke, 2001). O fluxo de umidade adicional resultante pode aumentar a umidade dentro da CLA e, dessa forma, torná-la termodinamicamente favorável ao aumento da chuva. No entanto, podem ocorrer situações opostas. O uso excessivo do solo pode levar ao aumento do albedo, e ocasionar redução da atividade convectiva e consequentemente da precipitação (Alpert \& Mandel, 1986).

Diante do exposto é evidente que o aumento e diversificação no uso da água e expansão de atividades agrícolas no semiárido brasileiro podem resultar em uma multiplicidade de impactos que exigem monitoramento ambiental contínuo da região.

Neste contexto, o modelo numérico RAMS (Regional Atmospheric Modeling System), na versão 8.0 foi utilizado neste trabalho para avaliar o impacto ambiental resultante da expansão agrícola na bacia hidrográfica do rio São Francisco em áreas do bioma caatinga. Efeitos das mudanças nos processos de troca de energia entre a superfície e a atmosfera na temperatura local são avaliados. A atuação de um vórtice ciclônico de altos níveis (VCAN) modulou a atividade convectiva no período analisado.

\section{Material e Métodos \\ Características da Área de Estudo}


A região foco deste estudo engloba as microrregiões de Petrolina e Juazeiro e foi selecionada por representar um dos mais dinâmicos polos de fruticultura irrigada do Nordeste, e contribuir de forma substancial para o desenvolvimento socioeconômico da região. A expansão agrícola em área de caatinga amplia a questão sobre a necessidade de monitoramento contínuo do impacto de mudanças ambientais de natureza antrópica e avaliação da influência nos processos atmosféricos e hidrológicos do Submédio São Francisco. A localização geográfica da represa de Sobradinho e os principais perímetros públicos de irrigação situados na área de estudo são mostrados na Figura 1. O clima é tipicamente semiárido e a vegetação predominante é do tipo caatinga hiperxerófila (Correia, 2001; Correia et al., 2006a).

A estação chuvosa tem início em novembro/dezembro e termina em março/abril (Ramos, 1975; Silva Aragão et al., 1997). O início do período chuvoso está associado à atuação de sistemas como o cavado de ar superior do Atlântico Sul, vórtices ciclônicos de altos níveis (Kousky \& Gan, 1981) e sistemas frontais austrais que penetram na Região Nordeste (Kousky, 1980). Ramos (1975) destaca que a frequência de ocorrência de precipitação é máxima no período da manhã (tarde) em Petrolina (nas áreas elevadas a oeste da cidade).

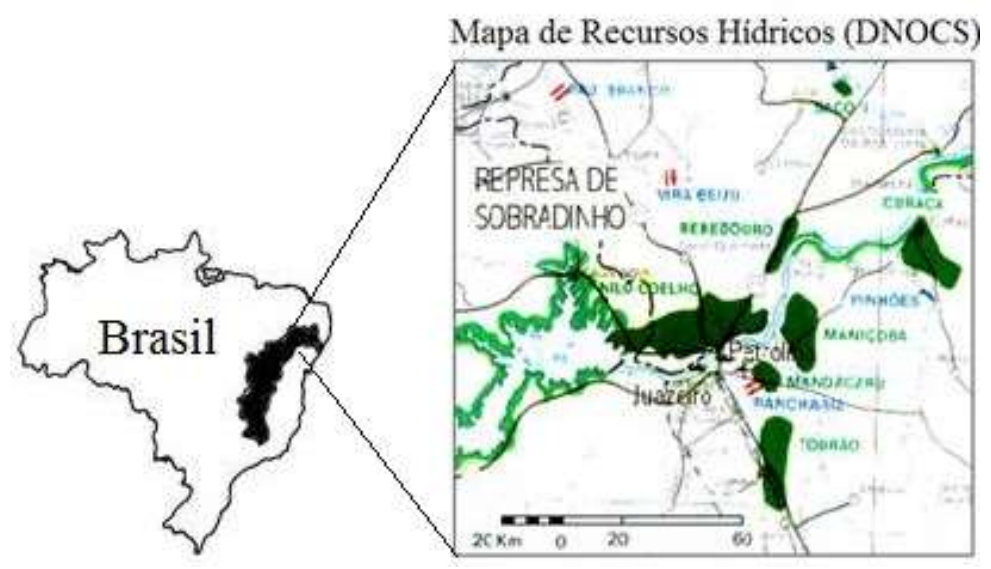

Figura 1. Mapa de Recursos Hídricos da região com uma visão parcial do lago de Sobradinho e dos perímetros públicos de irrigação no Submédio do Rio São Francisco. (Fonte: Modificado de Correia, 2001).

O autor sugere que esse comportamento é resultado da interação entre o escoamento de grande escala (ventos alísios) e a circulação local gerada pela desigualdade no aquecimento/esfriamento entre a área das encostas e do vale. A importância da topografia no desenvolvimento de circulações locais na região de Petrolina também foi evidenciada por outros pesquisadores. Correia et. al., (2006b), em um estudo numérico sobre o impacto ambiental da ocupação do solo na área de Sobradinho detectaram a importância do acoplamento entre a brisa lacustre e os ventos de encosta no transporte de umidade e calor na região.

As condições atmosféricas em Petrolina são caracterizadas pela presença de ventos do quadrante sudeste e de uma inversão de subsidência na baixa troposfera devido ao domínio da Alta Subtropical do Atlântico Sul (ASAS), que é mais intensa no final do outono e no inverno austral (Ramos, 1975). Essa inversão parece inibir a formação e desenvolvimento das nuvens mantendo a influência negativa na quantidade de precipitação. Entretanto, convecção profunda e precipitação intensa são comumente registradas no período chuvoso. A Zona de Convergência Intertropical, Vórtices Ciclônicos de Altos Níveis e, Sistemas Frontais austrais estão entre os principais sistemas que podem favorecer o desenvolvimento e intensificação de sistemas convectivos precipitantes (Silva Aragão et al., 2000; Souza, 2003; Diniz et al., 2004; Barbosa \& Correia,
2005; Silva Aragão et al., 2007; Santos Silva et al., 2008).

\section{Simulações Numéricas}

Simulações numéricas de alta resolução foram realizadas para quantificar o impacto ambiental da expansão da agricultura irrigada na variabilidade da temperatura local. Os experimentos foram configurados com duas grades aninhadas com resolução espacial de $8 \mathrm{~km} \mathrm{x} 2 \mathrm{~km}$, grades 1 e 2, respectivamente, ambas centradas em Petrolina-PE $\left(9,4^{\circ} \mathrm{S}-40,5^{\circ} \mathrm{W}\right)$. A grade vertical é constituída por 50 níveis com $\Delta \mathrm{z}$ inicial de 30 metros, aumentando para cima na razão de 1,1 até atingir $1 \mathrm{~km}$ de espessura. A partir desse nível, o $\Delta \mathrm{z}$ é constante até o topo do modelo $(22000 \mathrm{~m})$.

O modelo foi inicializado com condições de contorno lateral provenientes das reanálises do NCEP (National Centers for Environmental Predictions), disponibilizadas de 6 em 6 horas (00Z, 06Z, 12Z e 18Z) e com 17 níveis de pressão na vertical. Inicialmente, a especificação da topografia e da vegetação foi feita com base em arquivos de dados (resolução de $1 \mathrm{~km}$ ) obtidos por meio de radiômetros de altíssima resolução (Advanced Very High Resolution Radiometer-AVHRR) disponíveis para o modelo. Perfis verticais do déficit de pressão do vapor d'água (DPV) foram construídos com o propósito de 
avaliar a estratificação e distribuição vertical do vapor d’água.

\section{Construção de Cenários}

Os cenários utilizados neste estudo foram elaborados para representar condições ambientais nativas (sem influências antrópicas) e com alterações decorrentes da construção da represa de Sobradinho e da expansão agrícola em áreas de Caatinga. No processo de elaboração do cenário, considerou-se que a implantação da agricultura irrigada no Submédio São Francisco reflete simultaneamente os impactos do desenvolvimento econômico (crescimento agrícola) da região e da degradação do bioma. Neste contexto, os termos degradação ambiental e desmatamento foram usados neste trabalho tanto para representar a retirada da vegetação nativa normalmente usada para abastecimento dos fornos de carvoarias e olarias (lenha), como para a formação de áreas agrícolas pela substituição do bioma caatinga por culturas irrigadas ou de sequeiro.

Dois cenários foram elaborados para simulação dos processos atmosféricos antes e depois das mudanças antrópicas: o cenário sem alterações antrópicas denominado de "ctg.sl" no qual o domínio numérico é constituído predominantemente por vegetação nativa do tipo caatinga e sem a inclusão do lago de Sobradinho e o cenário atual, denominado de "ctg.pl.irg.cl" incluindo as mudanças antrópicas, em que a superfície no domínio numérico é representada por uma área com vegetação nativa (caatinga), plantações, culturas irrigadas e água (lago de Sobradinho). Os parâmetros biofísicos da caatinga usados no ajuste do esquema de superfície foram definidos com base em dados obtidos na literatura e coletados em experimentos de campo (Cunha, 2007; Correia et al., 2006).
Os arquivos de ocupação do solo foram gerados a partir de um programa desenvolvido em linguagem FORTRAN denominado de MUDVEG (Melo, 2011) que permite alterar informações existentes nos arquivos padrão do RAMS e construir cenários representativos das mudanças no bioma caatinga. O uso do MUDVEG permitiu substituir classes de vegetação existentes no default (padrão) do modelo por outros tipos selecionados conforme os objetivos de cada experimento numérico. No procedimento de inclusão e posicionamento dos perímetros irrigados utilizou-se inicialmente um mapa da distribuição espacial da vegetação proveniente de saídas numéricas realizadas com o modelo RAMS na versão 4a (resolução espacial de $2 \mathrm{~km}$ ) geradas em um estudo sobre impacto ambiental na área da Usina hidrelétrica de Sobradinho desenvolvido por Correia, (2001).

A distribuição dos principais perímetros públicos de irrigação no domínio numérico conforme assimilado pelo modelo é mostrado na Figura 2. As informações geográficas das áreas agrícolas usadas na construção dos arquivos dos dados de entrada no modelo foram extraídas de um mapa de recursos hídricos da região semiárida confeccionado pelo Departamento Nacional de Obras contra as Secas (DNOCS) no ano de 1989 (Figura 1). Uma visão conjunta da topografia e ocupação do solo no domínio numérico incluindo a vegetação padrão do RAMS e os cenários de controle, (sem mudanças antrópicas) "ctg.sl” e o cenário "ctg.irg.pl.cl” com mudanças antrópicas, pela inclusão do Lago de Sobradinho, áreas agrícolas e perímetros de irrigação é apresentada na Figura 3.

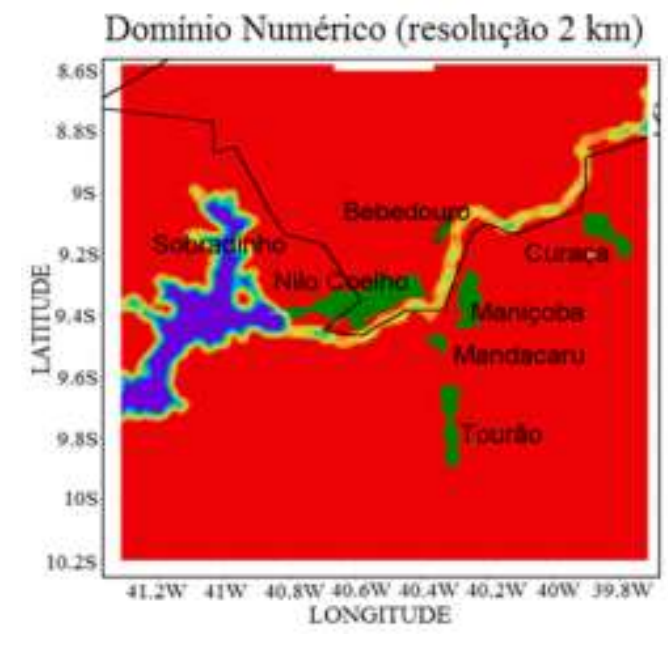

Figura 2: Domínio numérico coberto pela grade 2 com a distribuição das áreas irrigadas no domínio numérico conforme assimilado pelo modelo. (Fonte: Correia, 2001). 

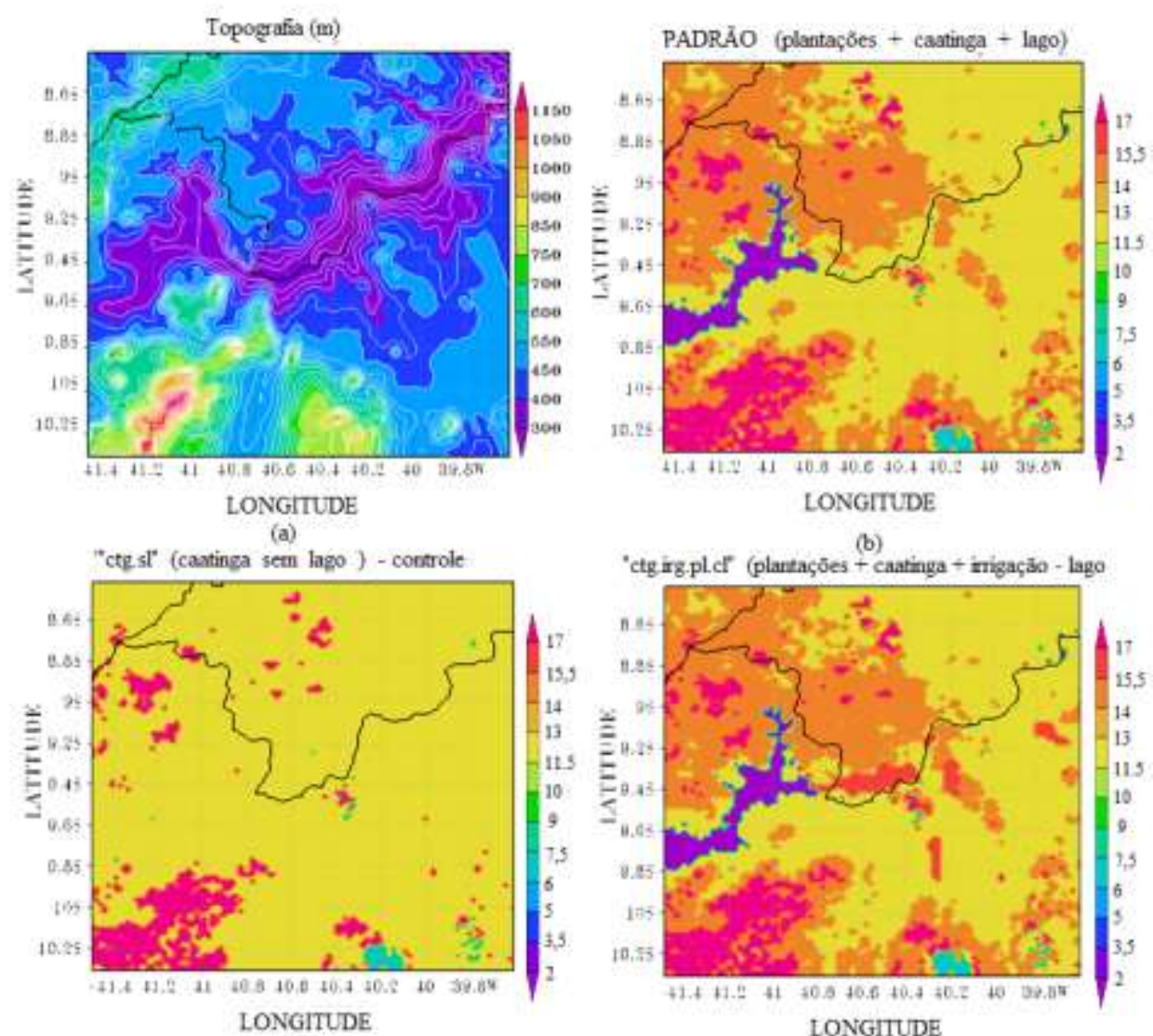

(c)

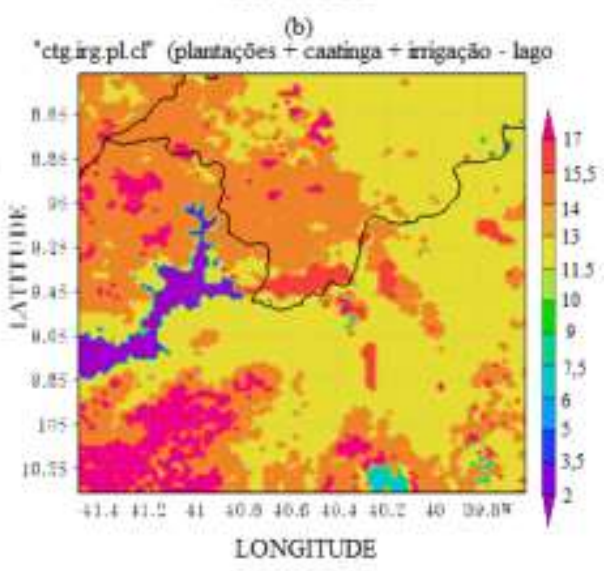

(d)

Figura 3: (a) Domínio numérico da grade 2: (a) Topografia; (b) vegetação padrão do RAMS; (c) ocupação do solo sem mudanças antrópicas, "ct.sl", e (d) ocupação do solo com mudanças antrópicas "ctg.irg.pl.cl”. A escala de cores indica as classes de vegetação: Lago (roxo), caatinga (amarelo), plantações (laranja), irrigação (vermelho) e cerrado (magenta).

\section{Resultados e Discussão}

Campos da distribuição espacial dos fluxos de calor sensível $(\mathrm{H})$ e latente (LE) resultante da diferença entre as simulações ctg.pl.irg.cl (com influências antrópicas) e ctg.sl (sem influências antrópicas) para os dias 14, 15 e 16 de março de 2005 , respectivamente, são apresentados na Figura 4. Os efeitos das mudanças na cobertura do solo são visíveis em toda a área coberta pelo domínio numérico. Nos dias 15 e 16 de março o impacto é mais nítido na região da represa de Sobradinho, e nas áreas agrícolas. Valores extremamente baixos resultantes da diferença entre as simulações mostram que na região inundada para formação do lago a redução no $\mathrm{H}$ foi de $200 \mathrm{~W} / \mathrm{m}^{2}$ no dia 15 e de $250 \mathrm{~W} / \mathrm{m}^{2}$ no dia 16 de março.

A diminuição no fluxo de calor sensível (H) também foi substancial nas regiões onde as plantas da Caatinga foram substituídas por agricultura irrigada atingindo valores da ordem $180 \mathrm{~W} / \mathrm{m}^{2}$ no dia 15 e de $250 \mathrm{~W} / \mathrm{m}^{2}$ no dia 16 de março (Figuras 4c,e). Especificamente na região do lago a queda nos valores de $\mathrm{H}$ é observada nos três dias (14, 15 e 16 de março) e pode ser explicada pelo aumento na disponibilidade de água na superfície (área inundada). Neste caso a maior parte da energia em superfície é usada para evaporar a água, reduzindo o fluxo de calor sensível.

Os resultados evidenciam que a variabilidade espacial dos fluxos turbulentos é bastante influenciada pelas características da superfície, topografia, disponibilidade de umidade no solo e resistência estomática das plantas. No entanto, a redução no teor de umidade da atmosfera normalmente referenciado como déficit de vapor da atmosfera, ou simplesmente como déficit de pressão de vapor (DPV) também representa um forte controle da taxa da evapotranspiração e pode afetar consideravelmente o balanço de energia em superfície e consequentemente a distribuição espacial dos fluxos de calor sensível $(\mathrm{H})$ e latente (LE). Perfis verticais do déficit de pressão do vapor d'água (DPV) para os dias 14, 15 e 16 de março construídos com base nos dados de pressões parciais do vapor d'água de saturação e do ar extraídos da simulação ctg.pl.irg.cl para Petrolina PE $(9,4 \mathrm{~S} ; 40,5 \mathrm{~W})$ são mostrados na Figura 4. Observa-se que no dia 16 de março a atmosfera apresenta um déficit de pressão de vapor bastante elevado, atingindo $18 \mathrm{hPa}$ em superfície. 


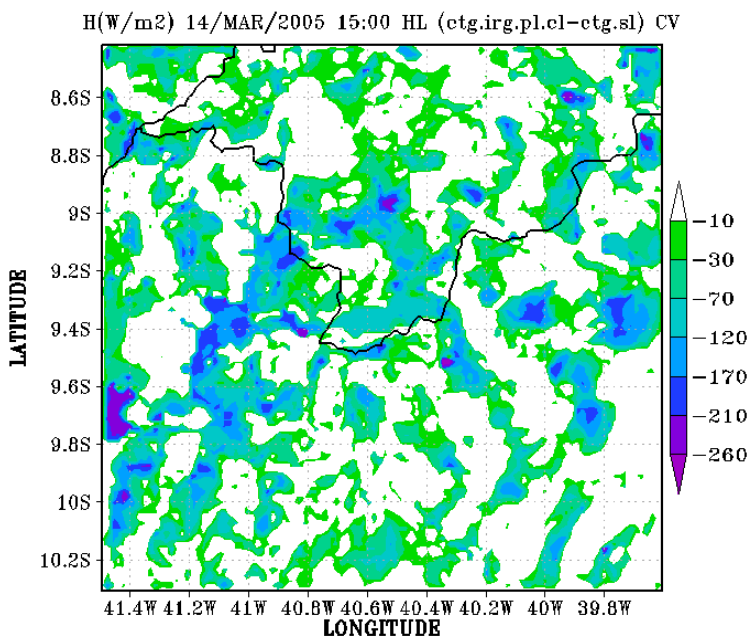

(a)

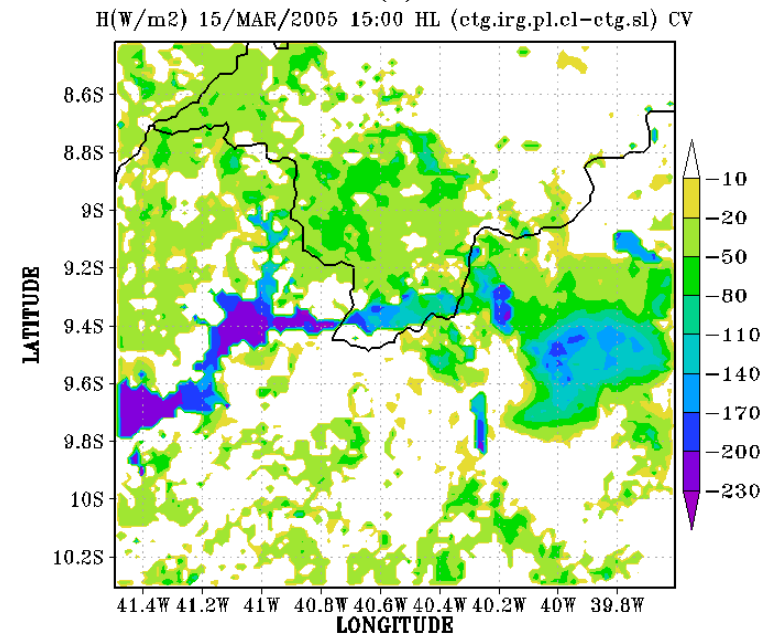

(c)

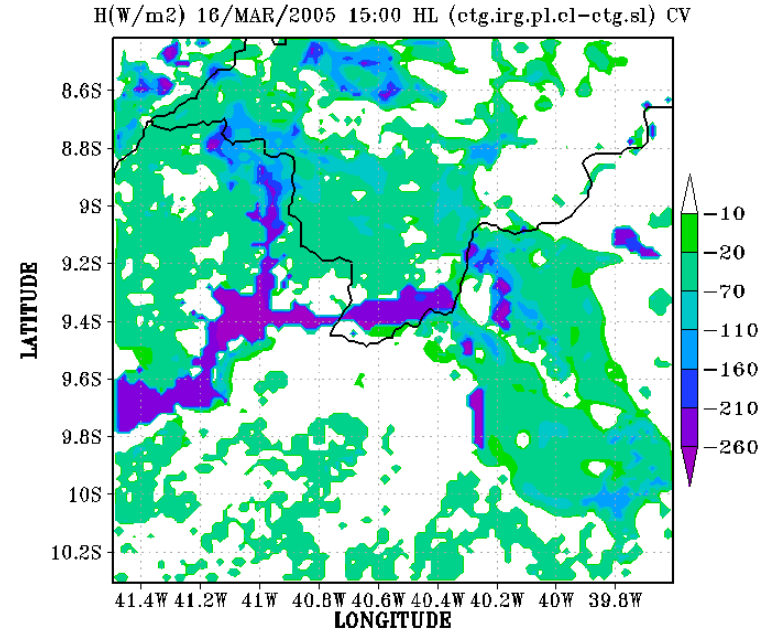

(e)

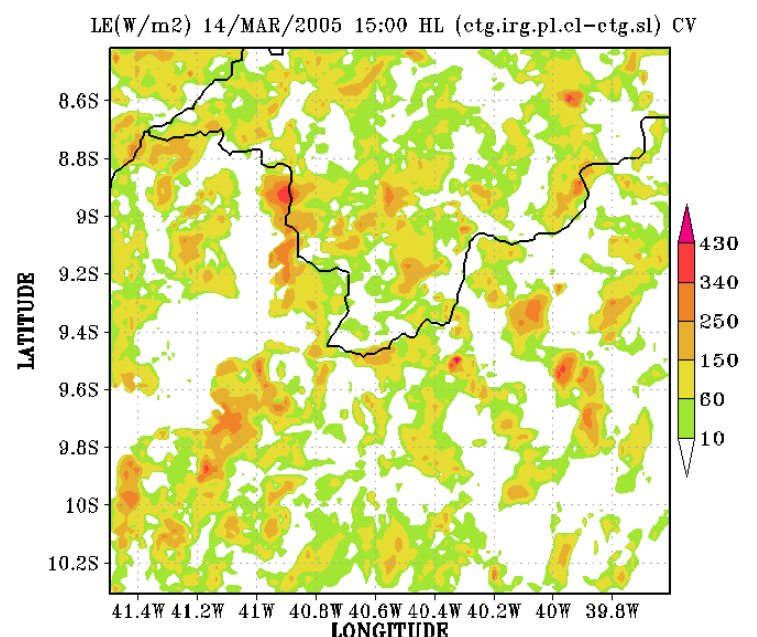

(b)

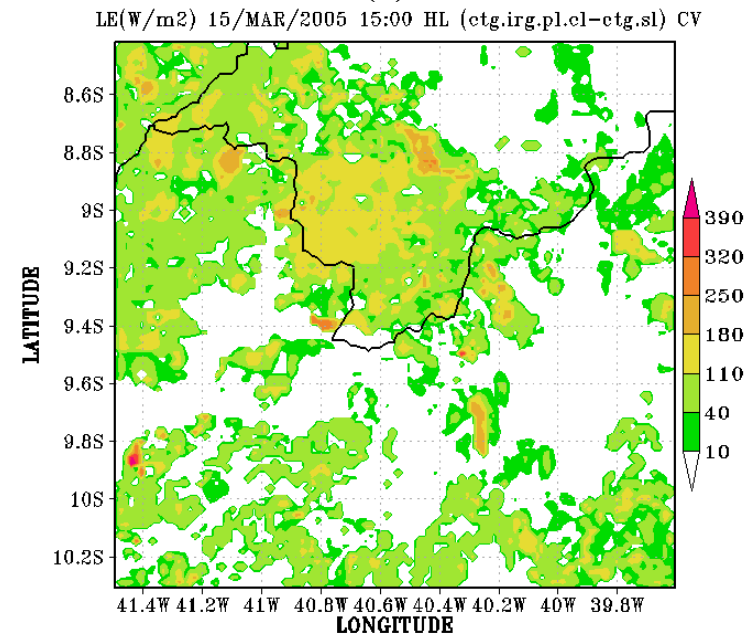

(d)

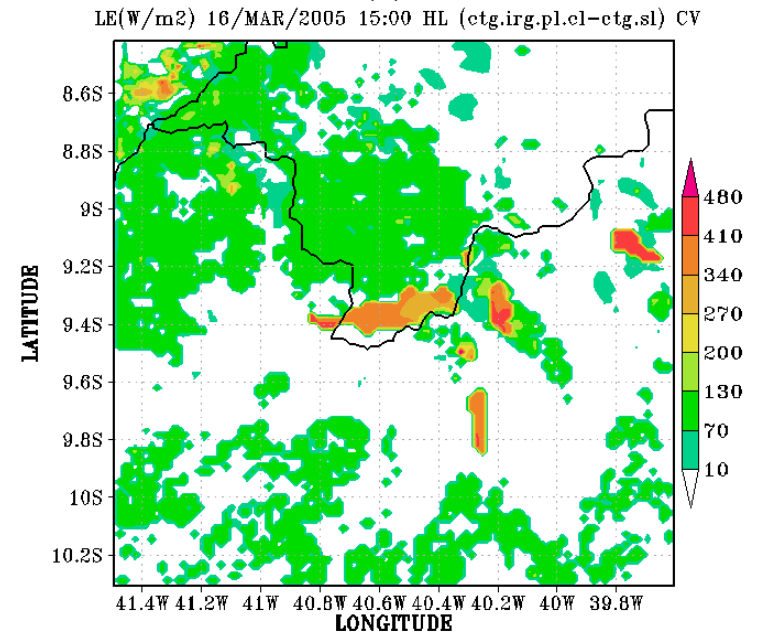

(f)

Figura 4. Distribuição espacial do fluxo de calor sensível $\mathrm{H}\left(\mathrm{W} / \mathrm{m}^{2}\right)$ nos dias: 14,15 e 16 de março (a), (c) e (e); e do calor latente $\mathrm{LE}\left(\mathrm{w} / \mathrm{m}^{2}\right)$ : nos dias: 14,15 e 16 de março (b), (d) e (f) obtida da diferença entre as simulações Caatinga, Culturas Irrigadas e Plantações com lago (ctg.irg.pl.cl) e Caatinga sem Lago (ctg.sl) as 15:00 HL.

A aparente organização dos fluxos $\mathrm{H}$ e LE, em faixas direcionadas no sentido SE/NW é um indicativo de que a topografia é um dos fatores que influenciam fortemente a distribuição horizontal e vertical dos fluxos de energia e água. A influência do terreno também é observada na região do Lago de Sobradinho (LS). A área inundada para formação do lago encontrase rodeada de terrenos elevados. Valores nitidamente mais altos de LE de aproximadamente $340 \mathrm{~W} / \mathrm{m}^{2}$ são observados a nordeste e ao sul do LS. Nestes setores 
tem-se o efeito conjunto do transporte de vapor pela brisa lacustre (BL) e pelos ventos de encosta (Melo, 2011).

O controle exercido pelo DPV (Figura 5) explica a grande diferença na configuração dos fluxos observada no dia 14 de março (Fig. 4a e b) quando comparada com a distribuição horizontal de $\mathrm{H}$ e LE observada nos dias 15 e 16 de março (Fig. 4c,d,e,f). A alta concentração de vapor na atmosfera no dia 14 de março reduz substancialmente a taxa de transpiração pelas

plantas.

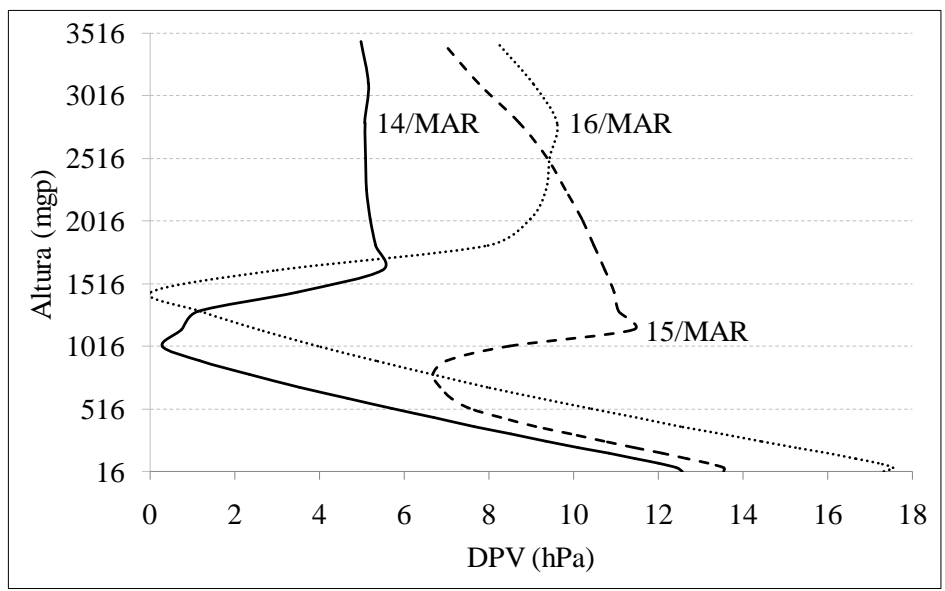

Figura 5: Perfis verticais do déficit de pressão do vapor d'água DPV para os dias 14, 15 e 16 de março de 2005. Os valores das pressões parciais do vapor d'água de saturação e do ar foram obtidos para Petrolina com base na simulação com o cenário atual (ctg.irg.pl.cl).

A mudança na configuração espacial dos fluxos nos dias 15 e 16 de março é um indicativo da redução no impacto da topografia em relação ao efeito de outros processos físicos decorrentes da variabilidade nas condições atmosféricas. Com o aumento no déficit de vapor da atmosfera o controle pelos estômatos no processo de evapotranspiração passa a ser dominante, modificando a partição da energia líquida disponível em $\mathrm{H}$ e LE.

Nos dias 15 e 16 de março, o impacto da substituição da vegetação nativa (Caatinga) por plantações e culturas irrigadas é mais evidente nos campos de $\mathrm{H}$ e LE. A queda nos valores de $\mathrm{H}$ resultante da diferença entre as simulações é bem acentuada, da ordem de $50 \mathrm{~W} / \mathrm{m}^{2}$ nas áreas de plantações e de 250 $\mathrm{W} / \mathrm{m}^{2}$ nas regiões dos perímetros irrigados e na área do Lago de Sobradinho.

Nos campos de LE o resultado da diferença entre as simulações ctg.irg.pl.cl e ctg.sl, mostra um aumento da evapotranspiração da ordem de $180 \mathrm{~W} / \mathrm{m}^{2}$ no dia 15 de março onde a vegetação nativa foi substituída por plantações (vegetação de sequeiro e pastagens) e de aproximadamente $380 \mathrm{~W} / \mathrm{m}^{2}$ no dia 16 de março nas regiões modificadas pela implantação dos perímetros irrigados.

Os valores obtidos para LE nos dias 15 e 16 de março mostram o efeito conjunto do teor de umidade no solo e do déficit de vapor na atmosfera nos processos de evapotranspiração. No dia 15 de março a diferença nos valores de LE pela substituição da Caatinga por vegetação irrigada e plantações não é tão evidente quanto no dia 16 (maior DPV) em que o efeito conjunto da grande quantidade de água no solo da região irrigada e da redução no teor de vapor da atmosfera aumenta consideravelmente a taxa de transpiração pelas plantas.

Esse resultado mostra também que a influência da mudança no uso da terra é determinante quando comparada com o impacto do tipo de vegetação na distribuição dos fluxos turbulentos. O impacto é maior nas regiões de vegetação irrigada decorrente da mudança no uso da terra (mudança no teor de umidade do solo). Efeitos semelhantes foram encontrados por Correia, et. al., (2006b).

\section{Conclusões}

As análises realizadas neste trabalho permitiram concluir que:

A quantidade de energia que atinge a superfície (radiação de onda curta e onda longa) é afetada pela estratificação de vapor na atmosfera e representa um fator limitante de grande impacto nos processos de evapotranspiração;

Os fluxos turbulentos simulados (H e LE) em períodos com maior atividade convectiva e precipitação não são tão sensíveis a irrigação. No entanto, em dias mais secos (maior déficit de vapor na atmosfera) são bastante influenciados pela disponibilidade de água no solo em áreas irrigadas. Esse efeito é mais pronunciado do que o impacto da mudança da vegetação nativa por áreas agrícolas. 


\section{Agradecimentos}

Aos revisores pelas sugestões. À Unidade Acadêmica de Ciências Atmosféricas/Universidade Federal de Campina Grande (DCA/UFCG).

Este trabalho é parte da pesquisa de doutorado desenvolvida pelo primeiro autor com o apoio financeiro do Conselho Nacional de Desenvolvimento Científico e Tecnológico $(\mathrm{CNPq})$ através do Projeto CLIMUD (Processo CNPq 504189/2003-4).

O primeiro autor agradece ao Programa de Pós-graduação em Meteorologia da Universidade Federal de Campina Grande (UFCG) e à Coordenação de Aperfeiçoamento de Pessoal de Nível Superior (CAPES) pela concessão de bolsa de estudos.

\section{Referencias}

Adegoke, J.O.; Pielke SR., R.; Carleton, A.M. 2007. Observational and modeling studies of the impacts of agriculture - related land use change on planetary boundary layer processes in the Central U.S. Agricultural and Forest Meteorology, v.142, n.2, p.203-215.

Adegoke, J.O.; Pielke, R.A.; EAsteman, J.; Mahmood, R.; Hubbard, K.G. 2003. Impact of irrigation on midsummer surface fluxes and temperature under dry synoptic conditions: a regional atmospheric model study of the U.S. high plains. Monthly Weather Review, v.131, p.556-564.

Alpert, P.; MandeL, M. 1986. Wind variability- an indicator for a mesoclimate in Israel. Journal of Climate and Applied Meteorology, v.25, n.12, p.1568-1576.

BARBOSA, T.F.; CORREIA, M.F. 2005. Sistemas convectivos intensos no semiárido brasileiro: o controle da grande escala. Revista Brasileira de Meteorologia, v.20, n.3, p.395-410.

Barnston, A.G.; Schickedanz, P.T. 2005. The effect of irrigation on warm season precipitation in the southern great plains. Journal of Climate and Applied Meteorology, v.23, n.6, p.865-888.

Correia, M.F. 2001. Impacto das ações antrópicas no clima do submédio do Rio São Francisco: um estudo numérico e observacional. 181 f. Tese (Doutorado em Meteorologia)-Universidade de São Paulo, São Paulo.

Correia, M.F.; Silva Dias, M.A. F. 2003. Variação do nível do reservatório de Sobradinho e seu impacto sobre o clima da Região. Revista Brasileira de Recursos Hídricos. v.8, n.1, p.157-168.
Correia, M.F.; Silva Dias, M.A. F.; Silva Aragão, M.R. 2006a. Soil occupation and atmospheric variations over Sobradinho lake área. Part One: An observational analysis. Meteorology and Atmospheric Physics, v.94, p.103-113.

Correia, M.F.; Silva Dias, M.A.F.; Silva Aragão, M.R. 2006b. Soil occupation and atmospheric variations over Sobradinho lake área. Part Two: A regional modeling study. Meteorology and Atmospheric Physics, v.94, p.115-128.

Cunha, A P.M.A. 2007. Calibração do "Simplified Simple Biosphere Model - SiB" para o bioma Caatinga do Nordeste brasileiro. Dissertação (Mestrado em Meteorologia)-Instituto Nacional de Pesquisas Espaciais, São José dos Campos.

Damião, M.C. 1999. Um estudo das condições atmosféricas em janeiros secos e chuvosos no semiárido do Nordeste do Brasil e sua relação com a chuva em meses posteriores na Região. $158 \mathrm{f}$. Dissertação (Mestrado em Meteorologia)Universidade Federal da Paraíba, Campina Grande.

Diniz, M.C.; Correia, M.F.; Silva Aragão, M.R. 2004. Convecção profunda no submédio do Rio São Francisco: uma avaliação das condições atmosféricas na área do aeroporto de Petrolina - PE. Revista Brasileira de Meteorologia, São Paulo, v.19, n.3, p.273-286, dez.

KOUSKY, V. 1980. Diurnal rainfall variation in northeast Brazil. Monthly Weather Review, v.108, n.4, p.488-498.

Kousky, V.E.; Gan, M.A. 1981. Upper tropospheric cyclonic vortices in the tropical South Atlantic. Tellus, v.33, p. 538-551.

Melo, E.C.S. 2011. Simulações Numéricas das Interações Biosfera-Atmosfera em Área de Caatinga: uma análise da expansão agrícola em ambiente semiárido. 135 p. Tese (Doutorado em Meteorologia) - Universidade Federal de Campina Grande, Campina Grande.

Pielke, R.A. 2001. Influence of the spatial distribution of vegetation and soils on the prediction of cumulus convective rainfall. Reviews of Geophysics, v.39, p.151-177.

Ramos, R.P.L. 1975. Precipitation characteristics in the Northeast Brazil dry region. Journal of Geophysical Research, v.80, n.12, p.1665-1678,

Silva Aragão, M. R., Correia, M. F.; Araújo, H. A. 2000. Characteristics of C-Band meteorological radar echoes at Petrolina, Northeast Brazil. International Journal of Climatology, v.20, p.279298.

Silva Aragão, M.R.; Correia, M.F.; Sobral, Z.R. 1997. Vento à superfície e chuva em anos contrastantes 
Revista Brasileira de Geografia Física, vol.07, n.05 (Número Especial- VIWMCRHPE), (2014) 960-968.

no submédio São Francisco. Boletim Climatológico, n.3, p.213-217.

Silva Aragão, M.R.; Damião Mendes, M.C.; Cavalcanti, I.F.A.; Correia, M.F. 2007. Observational study of a rainy January day in the Northeast Brazil semi-arid region: synoptic and mesoscale characteristics. Quarterly Journal of the Royal Meteorological Society, v.133, p.1127-1141.

Souza, P.P. 2003. Estudo da atuação de um sistema de latitudes médias no semiárido do nordeste brasileiro. 102 f. Dissertação (Mestrado em Meteorologia)-Universidade Federal de Campina Grande, Campina Grande.
Stolhgren, T.J.; Chase, T.N.; Pielke, R.A.; Kittels, C.F.; Baron, J.S. 1998. Evidence that local land use practices influence regional climate, vegetation, and stream flow patterns in adjacent natural areas. Global Change Biology, v.4, p.495-504.

Wallace, J.S.; Roberts, J.M.; Sivakumar, M.V.K. 1990. The estimation of transpiration from sparse dry land millet using stomatal conductance and vegetation area indices. Agricultural and Forest Meteorology, v.51, p.35-49. 